VOL. 66 (2002) [91-93]

\title{
A BEURLING ALGEBRA IS SEMISIMPLE: AN ELEMENTARY PROOF
}

\section{S.J. Bhatt and H.V. Dedania}

The Beurling algebra $L^{1}(G, \omega)$ on a locally compact Abelian group $G$ with a measurable weight $\omega$ is shown to be semisimple. This gives an elementary proof of a result that is implicit in the work of M.C. White (1991), where the arguments are based on amenable (not necessarily Abelian) groups.

Let $G$ be a locally compact Abelian group with Haar measure $\lambda$. A weight on $G$ is a meaurable function $\omega: G \longrightarrow(0, \infty)$ such that $\omega(s+t) \leqslant \omega(s) \omega(t)(s, t \in G)$. Then the Beurling algebra $L^{1}(G, \omega)$ consists of all complex-valued measurable functions $f$ on $G$ such that $f \omega \in L^{1}(G)$. It is a commutative Banach algebra with convolution product and with the norm $\|f\|_{\omega}:=\int_{G}|f(s)| \omega(s) d \lambda(s)$. The authors faced the problem of the semisimplicity of $L^{1}(G, \omega)$ in the investigation of the unique uniform norm property in Banach algebras $([1])$. It is shown in [5] that if $G$ is amenable, then there exists a continuous, positive, $\omega$-bounded character on $G$. Then Lemma 2 (below) quickly implies that $L^{1}(G, \omega)$ is semisimple for an Abelian $G$. Since the theory of amenable groups is not (yet) a standard part of Harmonic Analysis, and certainly not a part of Abelian Harmonic Analysis, we present an elementary proof of this basic result within the context of Abelian groups.

Theorem 1. The Beurling algebra $L^{1}(G, \omega)$ is semisimple.

LEMma 2. $L^{1}(G, \omega)$ is either semisimple or radical.

Proof: Assume that $L^{1}(G, \omega)$ is not radical. So its Gelfand space $\Delta\left(L^{1}(G, \omega)\right)$ is non-empty. Let $\varphi \in \Delta\left(L^{1}(G, \omega)\right)$. Then there exists a function $\alpha \in L^{\infty}(G, 1 / \omega)$, the Banach space dual of $L^{1}(G, \omega)$, such that

$$
\varphi(f)=\int_{G} f(s) \alpha(s) d \lambda(s)
$$

for all $f \in L^{1}(G, \omega)$. By the standard argument in the case of $L^{1}(G)$, one can show that $\alpha$ is a continuous function, $0<|\alpha(s)| \leqslant \omega(s)(s \in G)$ and $\alpha(s+t)=\alpha(s) \alpha(t)(s, t \in G)$.

Received 8th January, 2002

The authors are thankful to Professor H.G. Dales, Leeds (UK), for drawing their attention towards the paper [5].

Copyright Clearance Centre, Inc. Serial-fee code: 0004-9727/02 \$A2.00+0.00. 
For each $\theta \in \widehat{G}$, define $\alpha_{\theta}$ by

$$
\alpha_{\theta}(g)=\int_{G} g(s) \alpha(s) \theta(s) d \lambda(s), g \in L^{1}(G, \omega) .
$$

Then $\alpha_{\theta} \in \Delta\left(L^{1}(G, \omega)\right)$. Now let $f \in \operatorname{rad} L^{1}(G, \omega)$, the radical of $L^{1}(G, \omega)$. Then $\alpha_{\theta}(f)=\widehat{f}\left(\alpha_{\theta}\right)=\widehat{f \alpha}(\theta)=0 \quad(\theta \in \widehat{G})$. Since $f \in L^{\mathbf{1}}(G, \omega)$, we have $f \alpha \in L^{1}(G)$. Since $L^{1}(G)$ is semisimple and $\widehat{f \alpha}(\theta)=0(\theta \in \widehat{G})$, we have $f \alpha \equiv 0$ almost everhwhere on $G$. But $\alpha(s) \neq 0$ for any $s \in G$; and hence $f \equiv 0$ almost everywhere on $G$. This proves that $L^{1}(G, \omega)$ is semisimple.

LEMMA 3. Let $G_{1}$ be a locally compact Abelian group such that $L^{1}\left(G_{1}, \omega\right)$ is semisimple for every weight $\omega$ on $G_{1}$. Let $G_{2}$ be a locally compact Abelian group such that $L^{1}\left(G_{2}, \omega\right)$ is semisimple for every weight $\omega$ on $G_{2}$. Let $G=G_{1} \oplus G_{2}$ be the direct sum. Then $L^{1}(G, \omega)$ is semisimple for every weight $\omega$ on $G$.

Proof: Let $\omega$ be a weight on $G$. By Lemma 2, it is enough to prove that $L^{1}(G, \omega)$ is not radical. Let $U_{1}$ and $U_{2}$ be symmetric neighbourhoods of the identities in $G_{1}$ and $G_{2}$ respectively such that their closures are compact. Define $f=\chi_{U_{1} \times U_{2}}$, the characteristic function of $U_{1} \times U_{2}$. Then $f$ is a non-zero element of $L^{1}(G, \omega)$. It is clear that $f^{n}=\chi_{U_{1}}^{n} \chi_{U_{2}}^{n}$ for all $n \in \mathcal{N}$. It is enough to show that $\lim _{n \rightarrow \infty}\left\|f^{n}\right\|_{\omega}^{1 / n}>0$. So define

$$
\begin{array}{lll}
\omega_{1}(s)=\omega(s, 0)\left(s \in G_{1}\right) & \text { and } & \omega_{2}(s)=\omega(0, s)\left(s \in G_{2}\right) \\
m=\inf \left\{\omega_{1}(s): s \in U_{1}\right\} & \text { and } \quad M=\sup \left\{\omega_{2}(s): s \in U_{2}\right\}
\end{array}
$$

It is clear that $\omega_{i}$ is a weight on $G_{i}(i=1,2)$. Then by $[2$, Proposition 2.1], $m>0$ and $M<\infty$. Also note that for any $n \in \mathcal{N}, \omega_{2}(s) \leqslant M^{n}$ for all $s \in U_{2}+\cdots+U_{2}$ (n-times) and

$$
\begin{gathered}
\left\|f^{n}\right\|_{\omega}=\int_{G}\left|f^{n}(s, t)\right| \omega(s, t) d \lambda_{1}(s) d \lambda_{2}(t) \\
=\int_{G_{1}} \int_{G_{2}}\left|\chi_{U_{1}}^{n}(s)\right|\left|\chi_{U_{2}}^{n}(t)\right| \omega(s, t) d \lambda_{1}(s) d \lambda_{2}(t) \\
\geqslant \int_{G_{1}} \int_{G_{2}}\left|\chi_{U_{1}}^{n}(s)\right|\left|\chi_{U_{2}}^{n}(t)\right| \frac{\omega_{1}(s)}{\omega_{2}(-t)} d \lambda_{1}(s) d \lambda_{2}(t) \\
=\int_{G_{1}}\left|\chi_{U_{1}}^{n}(s)\right| \omega_{1}(s) d \lambda_{1}(s) \int_{G_{2}}\left|\chi_{U_{2}}^{n}(t)\right| \frac{1}{\omega_{2}(-t)} d \lambda_{2}(t) \\
\geqslant\left\|\chi_{U_{1}}^{n}\right\|_{\omega_{1}} \frac{1}{M^{n}} \int_{G_{2}}\left|\chi_{U_{2}}^{n}(t)\right| d \lambda_{2}(t) \\
=\frac{1}{M^{n}}\left\|\chi_{U_{1}}^{n}\right\|_{\omega_{1}}\left\|\chi_{U_{2}}^{n}\right\|_{1},
\end{gathered}
$$

where $\|\cdot\|_{1}$ denotes the $L^{1}$-norm and $\lambda_{i}$ denotes the Haar measure on $G_{i}$ for $i=1,2$. Then $\lim _{n \rightarrow \infty}\left\|f^{n}\right\|_{\omega}^{1 / n} \geqslant(1 / M) \lim _{n \rightarrow \infty}\left\|\chi_{U_{1}}^{n}\right\|_{\omega_{1}}^{1 / n} \lim _{n \rightarrow \infty}\left\|\chi_{U_{2}}^{n}\right\|_{1}^{1 / n}>0$. This proves that $L^{1}(G, \omega)$ is semisimple. 
Proof of Theorem 1: Note that if $G$ is a compact Abelian group, then $L^{1}(G, \omega)$ $=L^{1}(G)$ for any weight $\omega$ on $G$; so it is semisimple. By [3, p. 113], $L^{1}(\mathcal{R}, \omega)$ is semisimple for any weight $\omega$ on $\mathcal{R}$; so Lemma 3 implies that $L^{1}\left(\mathcal{R}^{n}, \omega\right)$ is semisimple for any weight $\omega$ on $\mathcal{R}^{n}$, where $n \geqslant 1$. Hence, again by Lemma $3, L^{1}\left(\mathcal{R}^{n} \oplus H, \omega\right)$ is semisimple for any weight $\omega$ on $\mathcal{R}^{n} \oplus H$, where $n \geqslant 0$ and $H$ is a compact Abelian group.

Now let $G$ be an arbitrary locally compact Abelian group and let $\omega$ be a weight on $G$. By [4, Theorem 2.4.1], there exists an open subgroup $G_{1}$ of $G$ such that $G_{1}=\mathcal{R}^{n} \oplus H$, where $n \geqslant 0$ and $H$ is a compact Abelian group. By above argument $L^{1}\left(G_{1}, \omega_{\mid G_{1}}\right)$ is semisimple. But the later is a closed subalgebra of $L^{1}(G, \omega)$. Hence $L^{1}(G, \omega)$ is not radical. Thus it is semisimple due to Lemma 2.

\section{REFERENCES}

[1] S.J. Bhatt and H.V. Dedania, 'Banach algebras with unique uniform norm II', Studia Math. 147 (2001), 211-235.

[2] H.A.M. Dzinotyiweyi, 'Weighted function algebras on groups and semigroups', Bull. Austral. Math. Soc. 33 (1986), 307-318.

[3] I. Gelfand, D. Raikov and G. Shilov, Commutative normed rings (Chelsea Publication Company, New York, 1964).

[4] W. Rudin, Fourier analysis on groups, Interscience Tracts in Pure and Applied Mathematics 12 (Interscience Publishers, New York, London, 1962).

[5] M.C. White, 'Characters on weighted amenable groups', Bull. London Math. Soc. 23 (1991), 375-380.

Department of Mathematics

Sardar Patel University

Vallabh Vidyanagar - 388120

Gujarat

India

e-mail: sjb@spu.ernet.in

haresh@spu.ernet.in 